\title{
L'ixeutique, un exemple de gestion empirique de la biodiversité végétale par la communauté traditionnelle Tsaaya du massif du Chaillu, Congo
}

The ixeutic, an empirical example of flora diversity management by the Tsaaya traditional community of Chaillu forest, Congo

Victor Kimpouni, Jean Pierre Makita-Madzou, Ernest Apani et Marcel Motom

\section{OpenEdition}

Journals

Édition électronique

URL : http://journals.openedition.org/ethnoecologie/1670

DOI : 10.4000/ethnoecologie.1670

ISSN : 2267-2419

Éditeur

Laboratoire Eco-anthropologie et Ethnobiologie

\section{Référence électronique}

Victor Kimpouni, Jean Pierre Makita-Madzou, Ernest Apani et Marcel Motom, «L'ixeutique, un exemple de gestion empirique de la biodiversité végétale par la communauté traditionnelle Tsaaya du massif du Chaillu, Congo », Revue d'ethnoécologie [En ligne], 5 | 2014, mis en ligne le 30 juin 2014, consulté le 01 mai 2019. URL : http://journals.openedition.org/ethnoecologie/1670 ; DOI : 10.4000/ ethnoecologie. 1670

\section{Ce document a été généré automatiquement le 1 mai 2019.}

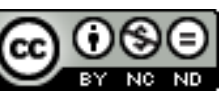

Revue d'ethnoécologie est mis à disposition selon les termes de la licence Creative Commons Attribution - Pas d'Utilisation Commerciale - Pas de Modification 4.0 International. 


\section{L'ixeutique, un exemple de gestion empirique de la biodiversité végétale par la communauté traditionnelle Tsaaya du massif $d u$ Chaillu, Congo}

The ixeutic, an empirical example of flora diversity management by the Tsaaya traditional community of Chaillu forest, Congo

Victor Kimpouni, Jean Pierre Makita-Madzou, Ernest Apani et Marcel Motom

\section{Introduction}

1 Les anthropologues occidentaux comme Lévi-Strauss (1955), Malinoswki (1968), EvansPritchards (1969), Lowie (1969) ont étudié et décrit les mœurs des sociétés traditionnelles et/ou secrètes des peuples qu'ils ont qualifiés de « primitives ». Décrivant les manières de chasser, de pêcher, de célébrer les fêtes profanes et sacrées, de cultiver, d'ensevelir les morts, nombreux d'entre eux, en particulier ceux des premiers âges de l'anthropologie, comme le dit expressément Evans-Pritchards (1969), tiraient la substance de leurs discours simplement à partir des informations, parfois biaisées, données par les autochtones rencontrés. Aussi, ils n'avaient point pratiqué les scènes qu'ils décrivaient et encore moins s'initier aux sociétés populaires ou secrètes, des indigènes dont ils révélaient les intimes valeurs. C'est ainsi que le rendu des savoirs endogènes de ces sociétés traditionnelles africaines réserve encore une bonne part des non-dits. En effet, les rapports biodiversité et savoirs empiriques endogènes sont encore partiellement explorés, comme le font observer Grenand \& Prévost (1994), Kimpouni \& Nguembo (2007) et Kimpouni et al. (2007) dans leurs travaux. 
2 Avant l'époque coloniale et au cours de celle-ci, l'homme a toujours cherché à améliorer son cadre de vie, en exploitant la biodiversité à portée de la main. L'exploitation des plantes à latex et à glu est une pratique séculaire de par le monde et des populations africaines, notamment celles du Bassin du Congo (Raponda-Walker \& Sillans 1961). Elle était destinée à la capture des oiseaux (ixeutique), la préparation des poisons de pêche et de chasse (flèches empoisonnées), et dans une moindre mesure la phytothérapie. Cette activité naguère sociale et clanique, devint de l'extractivisme au seul profit du colon. Ainsi, comme le montre les travaux de Lecomte (1900) cités par Raponda-Walker \& Sillans (1961) plusieurs plantes à latex et/ou à glu seront expérimentées pour la fabrication du caoutchouc «sauvages ou des herbes» (Hua 1907; Charles et al. 1969; Neuwinger 1996). Les meilleures potentialités étant trouvées chez Funtumia elastica, seule cette espèce fut alors exploitée intensivement (Raponda-Walker \& Sillans 1961). Les conséquences de cette période ont conduit plus d'une communauté traditionnelle, à perdre ses repères sociaux et avec eux toute l'expérience empirique accumulée au cours des âges. En Afrique subsaharienne, les données de Raponda-Walker \& Sillans (1961) sur l'ixeutique relèvent l'usage des mêmes espèces, productrices de latex et/ou de glu, au Gabon, en RD Congo et en Afrique de l'Ouest.

3 À l'opposé de ces chercheurs, nous donnons un compte rendu scientifique authentique de l'ixeutique chez les Tsaaya, telle qu'elle est pratiquée depuis toujours. En effet, initiés à l'ixeutique, nous l'avons pratiquée de façon effective, en accomplissant toutes les opérations, de la reconnaissance des plantes à glu dans les formations forestières à la capture des oiseaux englués.

4 Cette étude sur l'homme et son environnement, est le résultat des observations pertinentes portant sur le volet de l'ethnobotanique en général et plus particulièrement à l'inventaire de plantes utiles à la société traditionnelle Tsaaya et aux aspects technologiques. L'intérêt de consacrer ce travail aux connaissances techniques liées à l'ixeutique, porte sur le fait qu'elles sont le plus souvent considérées comme mineures devant les aspects alimentaires, médicinales et économiques de la biodiversité. Ce travail cadre avec la problématique de la valorisation et de l'amélioration des connaissances sur l'empirisme et les mécanismes d'exploitation et de gestion des bioressources, par les communautés traditionnelles.

\section{Présentations de la communauté Tsaaya et du site d'étude}

\section{Communauté Tsaaya}

5 La communauté ethno-linguistique Tsaaya est une entité du grand groupe ethnique Téké. Dupré (1972) signale leur existence sous le nom de «Bantchayi» et les désigne indifféremment, dans ses travaux d'ethnographie, par les termes de "Tékés forestiers » ou de «Tékés de l'ouest ». Comme toutes les communautés traditionnelles forestières du Congo, la société Tsaaya ne fait pas exception aux maux liés à la société moderne (exode rural, exploitation forestière et minière). Victime d'une déstabilisation sociale, la communauté ethno-linguistique Tsaaya perd les valeurs cardinales du fondement de son socle, suite au vieillissement de sa population. Les données démographiques du recensement de la population ne permettent pas de faire une estimation de n'importe 
quelle communauté traditionnelle. Fondées sur les entités administratives, elles n'intègrent pas la dimension ethnico-linguistique.

Les travaux de Bouquet (1969) et de Dupré (1972) montrent que les Tsaaya occupent actuellement une aire qu'ils partagent avec d'autres communautés forestières (Téké Laali, Nzabi, Tsangui, Mbamba, Yaka et Voumbou). Ce territoire qui s'étend jusqu'au Gabon, est limité au Congo aux circonscriptions administratives de Zanaga, Komono, Bambama et Mossendjo (Vennetier 1977). Traditionnellement agriculteurs et chasseurs, ils pratiquent encore le ramassage, la cueillette et la protoculture dans les formations végétales naturelles. L'ixeutique par contre, figure parmi les préoccupations les plus régulières, visant à diversifier les apports en nutriments carnés.

\section{Localisation du site d'étude}

7 Le massif du Chaillu est un des trois massifs forestiers congolais. Il se situe au sud-ouest et s'étale sur deux régions administratives à savoir le Niari et la Lékoumou (Fig. 1). Dans ce territoire les Tsaaya se rencontrent dans les districts de Komono, Bambama et Zanaga dans la Lékoumou et Mossendjo dans le Niari.

Figure 1. Localisation du terroir Tsaaya au sein du massif du Chaillu
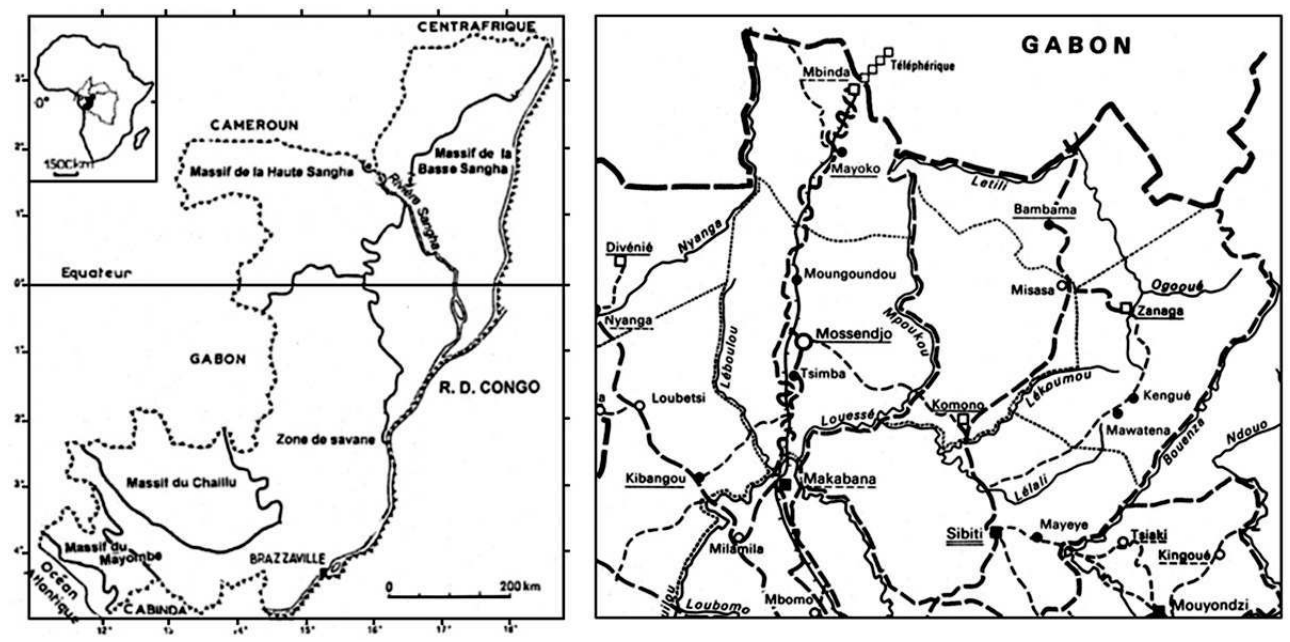

La zone d'étude présente un relief assez contrasté dont l'altitude varie de 400 à $800 \mathrm{~m}$. La formation végétale dominante est la forêt dense ombrophile semi-caducifoliée, se développant sous un climat qualifié de bas-congolais (Aubréville 1949; Vennetier 1977; Samba-Kimbata 1978). Il se caractérise par des précipitations d'environ $1800 \mathrm{~mm} / \mathrm{an}$ et des températures moyennes de l'ordre de $25^{\circ} \mathrm{C}$. La végétation est soumise à deux saisons très contrastées et de durée inégale. La saison de pluie, humide et chaude s'étale d'octobre à mai et accuse un ralentissement prononcé des précipitations de janvier à février. La saison sèche, marquée par l'absence de pluies, est fraîche et couvre la période allant de juin à septembre.

La population étant à majorité paysanne, les activités relatives à la production des biens alimentaires se limitent à l'agriculture itinérante sur brûlis, la chasse, la pêche et la cueillette. 


\section{Matériel et méthode}

10 L'enquête ethnobotanique se focalise sur la connaissance que les différents membres de la communauté Tsaaya expriment sur les vertus des plantes à glu et à latex. À propos du groupe d'informateurs, dont l'âge varie de 15 à plus de 60 ans, son socle se compose principalement des personnes ayant des connaissances prouvées sur cette activité (Tableau 1). Le nombre d'informateurs n'est pas fonction de l'importance de la localité en termes de résidents, mais plutôt en nombre d'individus possesseurs des secrets des plantes. C'est ainsi que 376 personnes ont été interrogées, dont 19 femmes ayant pratiqué l'ixeutique dans leur jeune âge, à côté des parents. Afin de suivre l'information et de la recouper, nous notons le groupe ethnique et l'origine de nos informateurs. Au cours de cet exercice, la démarche ethnobotanique consiste à identifier la ressource et à connaître les propriétés associées ou qui ont été associées. Enfin, cette phase est ponctuée par les opérations de recensement des espèces productrices de la glu et/ou du latex, la collecte et le traitement de la ressource dans la forêt naturelle. Cette procédure permet de sérier et d'authentifier l'information reçue et si possible de la matérialiser en relevant les signes tangibles d'exploitation.

Tableau 1. Tableau synoptique sur les caractéristiques du panel d'informateurs

\begin{tabular}{|c|c|c|c|c|}
\hline \multirow{2}{*}{ Tranche d'âge (ans) et genre } & \multicolumn{2}{|c|}{ Autochtones Tsaaya } & \multicolumn{2}{c|}{ Autres groupes ethniques } \\
\hline & Nombre & $\%$ & 18 & 85,7 \\
\hline $15-20$ & 255 & 76 & 2 & 9,5 \\
\hline $20-30$ & 61 & 18 & 1 & 4,8 \\
\hline 30 et plus & 20 & 6 & 21 & 5,6 \\
\hline Hommes & 336 & 89,4 & 0 & 0 \\
\hline Femmes & 19 & 5,1 & & $\%$ \\
\hline
\end{tabular}

11 Les enquêtes s'appuient sur les entretiens personnalisés et les « focus group » impliquant les deux genres. Les entretiens sont semi-directifs à questions ouvertes, afin de ne pas influencer la réponse attendue (Martin 1995).

12 Le matériel d'étude se compose des plantes spontanées, utilisées dans le territoire forestier du massif Chaillu. La taxinomie adoptée pour la classification dudit matériel est l'APG III (2009). La détermination des espèces animales a été faite par l'équipe de faunistique de la faculté des sciences de l'Université Marien Ngouabi.

\section{Résultats}

\section{L'ixeutique et le genre}

L'enquête sur l'ixeutique chez les Tsaaya révèle que cette activité est exclusivement masculine. Sa pratique est surtout l'œuvre des jeunes dont l'âge varie entre 15 et 20 ans. Toutefois, elle intéresse les femmes qui sont friandes des prises et informent sur les meilleurs acteurs. Les données ethnobotaniques mettent en évidence l'appropriation des connaissances empiriques des Tsaaya par les populations voisines. En effet, les 21 personnes non Tsaaya interrogées reconnaissent la pratique de l'ixeutique au sein de leur communauté respective et avouent la spécificité des techniques Tsaaya. 


\section{Diversité floristique et usages}

14 L'inventaire floristique ressort 15 espèces angiospermiennes se répartissant dans quatre familles (Tableau 2). Au plan morphologique, elles se présentent sous deux types distincts à savoir les arbres et les lianes. Parmi ces espèces on distingue les terrestres (les arbres et les lianes) et les épiphytes étrangleurs (Ficus thonningii). Les Sapotaceae avec 33,33\% sont les plus citées dans l'exploitation des vertus liées à l'ixeutique et des accessoires relatifs (appeaux). Des 15 espèces répertoriées, $20 \%$ produisent concomitamment le latex et la glu, alors que $46,67 \%$ donnent exclusivement du latex qui est transformé en glu par un traitement additionnel. Ce traitement additionnel est aussi valable pour le latex provenant des espèces pourvoyeuses de la glu brute.

Tableau 2. Diversité floristique en rapport à l'ixeutique et processus de collecte du latex et de la glu

\begin{tabular}{|c|c|c|c|}
\hline Taxons et diversité floristique relative & Parties utilisées & Types de produit & Modes de récolte \\
\hline \multicolumn{4}{|l|}{ Apocynaceae $(4$ / $26,67 \%)$} \\
\hline Clitandra cymulosa Benth. & tige & latex & saignée \\
\hline Funtumia africana (Benth.) Stapf & tige & latex & saignée \\
\hline Landolphia cf. dulcis (Sabine) Pichon & tige & latex / glu & Saignée / coupe \\
\hline Landolphia jumellei (Pierre ex Jum) Pichon & tige & latex & abattage (coupe) \\
\hline \multicolumn{4}{|l|}{ Arecaceae $(4 / 26,67 \%)$} \\
\hline Elaeis guineensis Jacq. & fruit \& feuille & Huile de palme gluau & extraction coupe \\
\hline Raphia hookeri Mann \& Wendl. & feuille & gluau & coupe \\
\hline Raphia regalis & feuille & gluau & coupe \\
\hline Raphia textilis & feuille & gluau & coupe \\
\hline \multicolumn{4}{|l|}{ Costaceae $(1 / 6,67 \%)$} \\
\hline Costus ligularis Bak. & tige & suc & coupe \\
\hline \multicolumn{4}{|l|}{ Moraceae $(1 / 6,67 \%)$} \\
\hline Ficus thonningii Blume & tige & latex / glu & saignée \\
\hline \multicolumn{4}{|l|}{ Sapotaceae $(5 / 33,33 \%)$} \\
\hline Chrysophyllum africanum A.DC. & fruit & latex / appeau & saignée / ramassage \\
\hline Chrysophyllum lacourtianum De Wild. & fruit & latex / appeau & saignée / ramassage \\
\hline Chrysophyllum pruniforme Pierre ex Engl. & fruit & latex / appeau & saignée / ramassage \\
\hline Omphalocarpum procerum P.Beauv. & fruit & latex / glu / appeau & saignée / coupe \\
\hline Synsepalum brevipes (Baker) T.D.Penn. & fruit & latex / appeau & saignée / ramassage \\
\hline
\end{tabular}

15 Quant à Elaeis guineensis et Costus ligularis, elles fournissent respectivement l'huile de palme et le suc (coagulum). Ces deux intrants, spécifient chacun un des deux processus conduisant à la transformation à froid du latex brut en glu. Notons que le troisième procédé de traitement additionnel est une réaction thermique, sans intrants.

\section{Importance des organes et exploitation}

Le latex tout comme la glu, est tiré de deux types d'organes dont un végétatif (la tige) et un génératif (le fruit). Des espèces impliquées, 50 \% le sont par l'organe végétatif et les autres par le fruit. Par contre, l'exploitation des Arecaceae et Costaceae dans le processus de l'ixeutique se base sur les propriétés de la tige chez Costus ligularus, du fruit d'Elaeis guineensis, de la feuille de Raphia sp. et Elaeis guineensis. Les graines des Sapotaceae évidées de leur amande et percées latéralement d'un orifice, servent d'appeaux.

\section{Processus de collecte du latex et/ou de la glu et des autres produits}

La récolte du latex et/ou de la glu est menée suivant le processus d'exploitation à faible impact. Les mécanismes de conservation de la glu baissent énormément la pression anthropique sur ces espèces, voire même sur Landolphia cf. dulcis, qui fait l'objet de coupe. 

catégorie. Les appeaux issus des graines de Sapotaceae sont acquis par ramassage. Cependant, la collecte du latex et de la glu découle de la saignée des organes végétatifs et génératifs, dans la majorité des cas. La coupe est systématique pour Landolphia cf. dulcis dont la liane n'excède pas $5 \mathrm{~cm}$ de diamètre. Quant aux fruits d'Omphalocarpum procerum, la saignée n'est valable que s'il est encore sur l'arbre. Le cas échéant, la collecte de la glu n'est possible que par la réalisation d'une coupe transversale ou longitudinale du fruit. Si l'huile de palme est obtenue des fruits d'Elaeis guineensis par extraction, les gluaux sont obtenus des nervures principales des folioles, alors que la tige de Costus ligularis est coupée et broyée pour en extraire le jus (suc).

\section{Saignées ou scarifications}

recéé s'applique aux lianes, aux troncs et aux fruits. Une saignée ou scarification est une incision oblique que l'on pratique jusqu'au niveau du cambium, pour les tiges. Elle permet l'obtention de la glu brute ou du latex, suivant les espèces impliquées.

Dans le cas des espèces donnant la glu brute, la coagulation du latex est rapide au contact de l'air. Il se forme une masse, qui prend régulièrement du volume, au niveau de l'incision. Ce saignement qui nous rappelle la formation des résines chez les Burseraceae ( Aucoumea klaineana, Canarium schweinfurthii, Dacryodes buettneri, Dacryodes klaineana) et Anacardiaceae (Mangifera indica), s'estompe au bout d'une heure environ, en formant une masse blanchâtre pouvant se répandre le long du tronc ou de la tige, voire au sol. Ce phénomène est observé chez Landolphia cf. dulcis, Clitandra cymulosa et Ficus thonningii. La collecte de la glu brute formée se réduit au prélèvement du " caillot ». Évidemment, il faut plusieurs scarifications pour accumuler une quantité suffisante de glu brute.

21 Pour le latex qui ne se prend pas au contact de l'air, donc reste fluide et s'écoule de l'organe au sol, il est recueilli suivant un processus de collecte identique à celui observé lors de la saignée de l'hévéa [Hevea brasiliensis (A.Juss.) Muell.Arg.]. Cette catégorie de latex est soumise à un traitement additionnel pour sa transformation en glu. Ces techniques sont obligatoires si l'on tient à exploiter les propriétés de Landolphia jumellei et Funtumia africana. La technique de récupération du latex après la scarification consiste à poser sous la saignée, un récipient dans lequel il tombe goutte à goutte ou en jet. On use souvent d'une calotte de calebasse [Lagenaria siceraria (Mol.) Standl.], d'une feuille de Marantaceae (Marantochloa holostachya (Bak.) Hutch., Marantachloa sp., Thaumatoccocus daniellii (Benn.) Benth.) ou de tout autre feuille pliée en cornet dit « kisuili ».

\section{Coupe de l'individu}

Consécutivement à son faible diamètre $(\leq 5 \mathrm{~cm})$, la technique de la coupe est systématique si l'on veut récolter le latex de Landolphia cf. dulcis et Clitandra cumulosa. Les conditions d'un bon rendement en latex sont liées au fait que la liane soit tranchée d'un coup sec, vu qu'elle ne tolère pas de bavures au niveau de l'écorce. La liane est ensuite sectionnée en morceaux de $0,5 \mathrm{~m}$ environ et placés sur un plan incliné de $45^{\circ}$ environ. La glu brute qui sourd se prend au contact de l'air, tout autour du cercle cambial. Après une journée ou deux au maximum on prélève la glu brute à la main. La récolte est rassemblée dans une calotte de calebasse, intérieurement ointe d'huile de palme ou en une boule que l'on enveloppe dans les feuilles de Marantaceae.

Revue d'ethnoécologie, 5 | 2014 


\section{Traitements additionnels du latex et de la glu brute}

Le latex ou encore la glu brute ne possède pas les qualités requises qui sont l'adhérence et la ténacité. C'est ainsi, qu'il faut les lui conférer par des préparations supplémentaires, suivant trois procédés soulignés dans la communauté Tsaaya.

- La première procédure consiste à mélanger le latex recueilli au jus (suc) de Costus ligularis. Pour des petites quantités de latex, mâcher la tige de Costus sp. et recracher la solution sur le latex. Il se prend rapidement en glu dès qu'il se mélange avec le jus de Costus ligularis. Il est intéressant de relever que toute la quantité de latex n'est pas transformée en glu, même si l'on augmente la quantité de jus (suc). Ce procédé n'est valable que pour le latex de Landolphia jumellei;

- La deuxième méthode conférant au latex les propriétés recherchées dans l'ixeutique, découle d'une réaction thermique. En effet, certains latex, notamment ceux qui ne réagissent pas au jus (suc) de Costus ligularis, se prennent une fois chauffés (porté au feu). Parmi les latex subissant ce traitement on a Funtumia africana;

- Enfin, le dernier procédé est relatif à la glu. Pour la rendre adhésive et tenace, la glu est mélangée à l'huile de palme (kilâma ma lihilini). Cette préparation est obligatoire pour toutes les glus. Afin de garantir le succès de cette opération, une précaution essentielle est à observer, l'huile de palme ne doit pas être en trop sinon la glu devient trop fluide, donc non tenace. Le bon dosage est d'environ $1 / 10$, soit une mesure d'huile de palme pour 10 de glu.

\section{Conservation de la glu}

Les Tsaaya conservent la glu de trois façons se déclinant comme suit :

- conservation au frais dans la maison: la glu est dans une calotte de calebasse (Lagenaria siceraria) ou dans un entrenœud de bambou (Bambusa vulgaris Schard. ex Wendl.) et le cas échéant empaquetée dans des feuilles et placée dans un endroit frais ;

- emballée dans les feuilles de Marantaceae et placée dans une portion de stipe de bananier, au sein du jardin de case ;

- emballée dans les feuilles de Marantaceae, elle est enfouie dans la vase humide.

\section{Spécificité des parties de chasse}

Les principales techniques de l'ixeutique chez les Tsaaya se résument à cinq systèmes, se différenciant suivant les conditions du milieu et la nature des éléments extrinsèques en présence.

- Kinab'sa: pratique très simple, elle consiste à s'armer d'une gaule de 3 à $5 \mathrm{~m}$ de long surmontée d'un gluau. Il est rabattu sur l'oiseau, en s'approchant à pas feutrés. Les captures sont constituées souvent d'oiseaux de petite taille (Souimanga chloris, Souimanga chloripygia, Astrilda mozambicus, Astrilda astrild, Astrilda melopoda, Serinus mozambicus, Colius striatus, Andropadus virens, Andropadus gracilis, Andropadus curvirostris, Prinia leucopogon, Dendropicos spp., Mesopicos spp., Anthreptes collaris, Nectarinia olivacea, Nectarinia cyanolaema, Nectarinia chloropygia, Nectarinia spp., Platysteria custanea, Platysteria cyanea, Merops gularis, Merops spp.). Notons que pendant la phase d'approche, la formule consacrée et récitée par le chasseur est "likete kuata, liketa kuata, ...». Littéralement cette expression se traduit par " engourdissement saisis-le, engourdissement saisis-le, ....»; au cours de cette partie de chasse, certains oiseaux ne se laissent jamais prendre. Tel est le cas de Pycnonotus barbatus. 
- Kikahama: le vocable kahama désigne les fourmis magnans (Dorylus sp.). Cette partie de chasse est tout à fait occasionnelle et opportuniste, vu qu'elle dépend des fourmis magnans. En effet, lorsqu'elles investissent un milieu à la recherche de nourriture, leur ligne de front peut s'étendre sur une vingtaine de mètres de largeur voire plus et une hauteur avoisinant les $2,5 \mathrm{~m}$ (hauteur du sous-bois). La férocité des magnans est telle que rien ne leur résiste sur le passage, même pas l'homme. Les oiseaux insectivores en particulier s'activent pour chasser les insectes en mouvement. Ces oiseaux qui suivent la progression des magnans recherchent toujours des perchoirs. C'est à ce moment qu'intervient le piégeur qui fraie à 10-20 $\mathrm{m}$ de la ligne de front des fourmis, une allée perpendiculaire au sens de leur progression. Il plante obliquement par rapport au sol des longues baguettes des tiges défeuillées en guise de perchoirs artificiels. La surface supérieure desdites tiges est enduite d'une couche de glu. Ce piège passif englue tout volatile qui s'y pose. Les oiseaux souvent englués sont: Pycnonotus barbatus, Merops spp., Astrilda spp., Andropadus spp., Ploceus spp., etc.;

- Yogo: pendant les périodes de forte chaleur, notamment aux mois de mars - avril et de septembre - octobre, les oiseaux vont se rafraîchir dans les petites trainées d'eau, les mares voire les abords des lacs ou le long des petits cours d'eau. Le piégeur profite de cette situation climatique exceptionnelle pour placer ses gluaux à l'endroit où les oiseaux viennent se baigner. Posés obliquement sur un support ou à même le sol par rapport à la grève, ils constituent des pièges passifs à tout oiseau qui s'y pose pour la séance de toilettage du plumage. Les captures se composent généralement de Ploceus spp., Vidua macroura, Astrilda mozambicus, Astrilda astrild, Astrilda melopoda, Serinus mozambicus, Colius striatus, Andropadus virens, Andropadus gracilis, Andropadus curvirostris, Bleda spp., Ixonotus guttatus, ... ;

- Kisuesuege : technique de chasse très commune en zone forestière. Le piégeur construit une hutte ronde de branchage et de feuilles. Il fraie une allée de quelques mètres de long pour moins de $0,7 \mathrm{~m}$ de large, sous futaie et y place un long gluau. Tapi dans la hutte, il souffle dans un appeau pour attirer les oiseaux aux alentours. Cette partie de chasse est celle qui fait courir au piégeur des risques insoupçonnés. En effet, blotti dans sa hutte, le ballet des oiseaux attire serpents et panthères en quête de pitance. Les oiseaux qui sont souvent capturés sont Andropadus virens, Andropadus gracilis, Andropadus curvirostris, Pycnonotus barbatus, Chlorocychla sp., Tauraco persa, ... ;

- Gluaux au nid et sur les aliments : procédé très simple, les gluaux sont placés sur le nid abritant les œufs ou les oisillons d'une part, et d'autre part sur les fruits mûrs sur pied comme la papaye (Carica papaya), la banane (Musa x sapientum) et régimes de noix de palme ( Elaeis guineensis). Les oiseaux souvent englués sont Colius striatus, Pycnonotus barbatus, Ploceus nigerrimus, Ploceus cucullatus, Vidua macroura, etc.

\section{Discussions et commentaires}

\section{Analyse ethnosociologique}

L'étude sur l'ixeutique chez les Tsaaya met en évidence une répartition des tâches, en fonction du genre. Comme c'est le cas dans la plupart des communautés traditionnelles forestières, le ravitaillement de la famille en produits carnés incombe au chef de ménage. Quant à la femme, sa responsabilité se limite à pourvoir le ménage des feuilles-légumes, des condiments et des produits agricoles, de cueillette et de pêche. Outre cet aspect de la répartition des travaux liée au sexe, nous avons pu mettre en évidence une appropriation et un partage des savoirs entre communautés voisines. 


\section{Analyse ethnobotanique} latex qui après un traitement additionnel donne la glu. Comme le montrent les travaux de Raponda Walker \& Sillans (1961), Profizi et al. (1993) et Kimpouni \& Nguembo (2007), toutes les communautés forestières vivant dans leur aire de répartition exploitent les vertus de ces plantes pour les mêmes fins. Cette étude sur l'ixeutique montre que les Tsaaya, en plus des Apocynaceae et des Moraceae connues de toutes les communautés traditionnelles forestières pour leur propriété inhérente à l'ixeutique, partagent avec les communautés du Nord Congo (Mboko, Bakuele, Bekwil), les vertus d'Omphalocarpum procerum. Cependant, ils sont les seuls à exploiter le latex de Chrysophyllum sp. Il en découle que l'usage de ces plantes en ixeutique appelle des connaissances endogènes qui sont divergentes, d'une communauté traditionnelle à l'autre. Relevant de l'empirisme, ces acquis sont des valeurs intrinsèques formant le socle des sociétés traditionnelles (Kimpouni 2001; Kimpouni et al. 2011, 2012). Dans le cas des Tsaaya, la classification des latex à glu suivant les espèces et le traitement additionnel spécifique à chacun d'eux sont des différences qui authentifient cette communauté. L'usage et la connaissance des adjuvants et des effets thermiques dans la stabilisation, la fixation, la précipitation des produits, sont des savoirs séculaires des communautés traditionnelles, à travers le monde (Grenand \& Prévost 1994). Cette étude des produits liés à l'ixeutique révèle que les Tsaaya sont la seule communauté traditionnelle usant des adjuvants [jus de Costus ligularis, de l'huile de palme (Elaeis guineensis)] et maitrisant la notion de dosage dans les mélanges. Par rapport aux autres communautés, ce particularisme tenant aux procédures techniques serait le plus qui leur permet d'utiliser une gamme plus large de plantes, et de reconnaitre que la composition chimique du latex varie d'une plante à l'autre.

Au regard de l'investissement en temps et l'analyse des marques sur les arbres et lianes, les taxons les plus fréquemment recherchés pour une application en ixeutique par les Tsaaya sont Landolphia cf. dulcis (Mutsia), Landolphia jumellei (Munzogo), Clitandra cymulosa (Kikeye) et Ficus thonningii (Litsana). Viennent ensuite Funtumia africana (Mudaana) et diverses espèces de Sapotaceae (Chrysophyllum sp., Omphalocarpum procerum, Synsepalum brevipes). Cette classification tient non seulement des propriétés physicochimiques de la glu brute obtenue ou celle résultant du traitement additionnel de celle-ci, mais aussi des difficultés inhérentes à la récolte. En effet, eu égard à la morphologie de la tige, les lianes se prêtent mieux aux saignées et à la coupe que les arbres. Ces derniers étant terrestres ou épiphytes requièrent des aptitudes à grimper pour atteindre les branches encore intactes voire moins incisées. Cet exercice, parfois périlleux, est un facteur majeur limitant leur exploitation.

Du point de vue de la qualité, les Tsaaya ont une préférence pour la glu de Landolphia cf. dulcis, Ladolphia jumellei, Clitandra cymulosa et Ficus thonningii. La collecte sur les autres espèces (Funtumia africana, Omphalocarpum procerum et Synsepalum brevipes) n'étant qu'occasionnelle, elle n'intervient qu'en cas de pénurie des premières citées.

Probablement en rapport avec les différentes méthodes de chasse, notamment le kikahama qui est une chasse opportuniste, les Tsaaya ont développé des modes de conservation de la glu récoltée ou préparée. Cette conservation de la glu couplée à la saignée des arbres ou des lianes constitue un mécanisme de gestion rationnelle et durable des espèces et des écosystèmes. Le point commun des trois techniques de conservation de

Revue d'ethnoécologie, 5 | 2014 
la glu, met en valeur les conditions ambiantes du milieu, notamment l'humidité. Elle paraît être l'élément déterminant qui évite la déshydratation qui induit le durcissement de la glu et donc la perte des propriétés physicochimiques, essentielles à la pratique de l'ixeutique.

31 Les techniques de chasse chez les Tsaaya révèlent des spécificités, propres à cette société traditionnelle. À la différence des autres communautés qui repèrent au préalable un endroit fréquenté par les oiseaux et enduisent les branches des arbres servant de perchoirs de glu, les Tsaaya mettent en place un astucieux arsenal de gluaux qu'ils peuvent mouvoir à tout instant et adapter aux circonstances du milieu.

\section{Conclusion}

Les plantes utilisées en ixeutique au Congo et dans les pays du Bassin du Congo sont nombreuses et très variées. Les techniques de collecte de la glu sont presque similaires dans toutes les contrées et au sein des communautés traditionnelles. La rentabilisation de cet acquis n'est possible que si les garants des valeurs sociales, possesseurs des savoirs endogènes et de leur perpétuation de génération en génération, assurent efficacement leurs rôles.

De cette étude, il se dégage que la communauté Tsaaya, du massif du Chaillu, est celle qui exploite au maximum les propriétés de la biodiversité végétale liées à l'ixeutique. Cette observation est d'autant plus plausible dans la mesure où une insuffisance de données ethnobotaniques et de botanique économique dans le domaine est relevée. À ce titre, une approche pluridisciplinaire donnerait une plus-value à cette étude. En effet, les différences notées sur le comportement du latex vis-à-vis des adjuvants peuvent être affinées, afin de valoriser les acquis du savoir traditionnel. Celle-ci devrait s'inscrire comme le soulignent Grenand \& Prévost (1994) dans «...une authentique valorisation $d u$ savoir de populations concernées, base préalable de la diffusion vers d'autres cultures. ... soutenir, parallèlement à nos recherches les revendications économiques et politiques des peuples qui nous communiquent leurs savoirs, en particulier en stimulant l'élaboration d'une législation internationale protégeant leurs droits de découvreurs.».

Nous exprimons toute notre gratitude aux membres de l'Équipe de faunistique de la faculté des sciences de l'Université Marien Ngouabi, notamment au Dr Grillot ${ }^{\dagger}$, pour sa laborieuse collaboration dans la partie ornithologique. Au Dr G. Mbani, pour le volet entomologie. Que la communauté Tsaaya soit ici honorée pour l'accueil et la générosité qui l'a caractérisée tout au long de cette étude.

\section{BIBLIOGRAPHIE}

APG III 2009 - An update of the Angiosperm phylogeny group classification for the orders and families of flowering plants: APG III. Botanical Journal of the Linnean Society 161: 105-121. 
Aubréville A. 1949 - Climats, forêts et désertification de l'Afrique centrale. Paris, Société d'éditions géographiques, maritimes et coloniales, $351 \mathrm{p}$.

Charles G., Njimi T., Ourisson G., Ehrhardt J.D., Conreur C., Cavé A. \& Goutarel R. 1969 - Alcaloïdes stéroïdiques 9 : Stéroïdes et alcaloïdes des graines de Funtumia elastica et de F. latifolia. C.r. 268 : 2105-2108.

Dupré M.-C. 1972 - La dualité du pouvoir politique chez les Téké de l'ouest : pouvoir Tsaayi et pouvoir Nzinéké (République Populaire du Congo). Thèse de $3^{\mathrm{e}}$ cycle, Ethnologie, Université de Lyon 2, $194 \mathrm{p}$.

Evans-Pritchard E. 1969 - Anthropologie sociale. Paris, Petite Bibliothèque Payot (Sciences de l'Homme), $177 \mathrm{p}$.

Grenand P. \& Prévost M.-F. 1994 - Les plantes colorantes utilisées en Guyane française. JATBA ( $n^{\text {elle }}$ série) 36 (1) : 139-172.

Hua H. 1907 - Apocynacées productrices de caoutchouc du Gabon septentrional recueillies par le Dr Gravot (Missions cottes). Bulletin du Muséum : 448-451.

Kimpouni V. 2001 - Contribution aux études ethnobotanique et floristique de la forêt de Lossi (Nord Congo) : Plantes de cueillette à usage alimentaire. Systematics and Geography of Plants 71: 679-686.

Kimpouni V., Apani E. \& Motom M. 2011 - Plantes ichtyotoxiques et particularisme des usages au Congo (Brazzaville). International Journal of Biological and Chemical Science 5 (3): 979-990.

Kimpouni V., Koubouana F., Apani E., Motom M. \& Makita-Madzou J.P. 2012 - Contribution à l'inventaire et à l'utilisation des plantes à effets psychotropes et toxiques au Congo (Brazzaville). Springer-Verlag France, DOI 10.1007/s10298-012-0684-8, Phytothérapie $10: 19-24$

Kimpouni V., Makita-Madzou J.P. \& Motom M. 2007 - Spécificités ethnoculturelles sur les produits d'Irvingia gabonensis et Irvingia spp. (Irvingiaceae) au Congo-Brazzaville. Annales de l'Université Marien Ngouabi 8(4) : 50-58.

Kimpouni V. \& Nguembo J. 2007 - Aspects d'exploitation et d'utilisation artisanale des produits forestiers d'origine végétale à Lossi (Congo Brazzaville), Communication présentée au $18^{\mathrm{e}}$ Congrès de l'AETFAT, du 26 février au 2 mars 2007, Yaoundé, Cameroun.

Levi-Strauss C. 1955 - Tristes tropiques. Paris, Plon, 456 p.

Lowie R.H. 1969 - Traité de sociologie primitive. Paris, Petite bibliothèque Payot, 443 p.

Malinowski B. 1968 - Trois essais sur la vie sociale des primitifs. Paris, Payot, 414 p.

Martin G.J. 1995 - Ethnobotany: A methods manual. London, Chapman \& Hall, 268 p.

Neuwinger H.D. 1996 - African ethnobotany: poisons and drugs - chemistry, pharmacology, toxicology. London, Chapman \& Hall, 941 p.

Profizi J.-P., Makita-Madzou J.-P., Milandou J.C., Karanda C.N., Motom M. \& Bitsindou I. 1993 Ressources végétales non ligneuses des forêts du Congo. Plan d'Action Forestier Tropical du Congo (PAFTCongo). Brazzaville, Université Marien Ngouabi, 67 p. + annexes.

Raponda-Walker R.A. \& Sillans R. 1961 - Les plantes utiles du Gabon. Paris, Lechevalier P., 614 p. Samba-Kimbata M.-J. 1978 - Le climat bas-congolais. Thèse de 3e cycle, Université de Dijon.

Vennetier P. 1977 - Atlas de la République Populaire du Congo. Paris, Jeune Afrique, 64 p. 


\section{RÉSUMÉS}

Les sociétés traditionnelles et surtout leurs savoirs ont intéressé plus d'un anthropologue, notamment ceux des premiers âges. En dépit des efforts consentis, les savoirs endogènes et empiriques des populations qualifiées de primitives, dans le domaine des études de l'homme et son environnement nous réservent encore des secrets. L'objet de cette étude est de mettre en évidence les savoirs des populations Tsaaya du Chaillu à travers l'ixeutique. Pratique séculaire de par le monde, l'ixeutique des Tsaaya se singularise par des (i) techniques connues nulle part ailleurs de transformation à froid du latex en glu, (ii) la conservation de celle-ci pour les parties de chasse futures et (iii) la fabrication d'instruments de chasse comme les appeaux. Cette étude ethnobotanique apporte des informations sur (i) les espèces de plantes impliquées dans l'ixeutique, (ii) les oiseaux souvent englués, (iii) et les mécanismes de traitement de collecte des produits et (iv) les types de chasse. Elle ressort 15 espèces spontanées (Clitandra cymulosa, Ficus thonningii, Funtumia africana, Landolphia of dulcis, Landolphia jumellei, Omphalocarpum procerum, Chrysophyllum africanum, Chrysophyllum lacourtianum and Synsepalum brevipes) et environ une trentaine d'espèces d'oiseaux. Quant aux appeaux, plusieurs espèces de Sapotaceae dont on extrait l'amande des graines sont recensées. Au niveau écologique et conservation de la biodiversité végétale, les techniques de récolte et de conservation de la glu sont décrites et analysées. l'ixeutique chez les Tsaaya est un exemple de gestion de la biodiversité. Cette étude s'inscrit dans la valorisation des savoirs Tsaaya liés à la gestion durable de la diversité floristique.

The traditional societies and especially their knowledge interested more than one anthropologist, in particular those of the first ages. In spite as of authorized efforts, the endogenous and empirical knowledge of the populations qualified of primitives, the field of the people and his environment studies still holds secrecies for us. The object of this study is to highlight the knowledge of the Tsaaya community of Chaillu through the ixeutic. Secular practice all over the world, the ixeutic of Tsaaya is made conspicuous by the (i) technical known ones nowhere elsewhere of cold transformation of latex into lime, (ii) the product conservation for the future hunting parties and (iii) the manufacturing of instrument of hunting like the bird calls. This ethnobotanic study brings information on (i) the plant species implied in the ixeutic one, (ii) the birds often limed and (iii) the mechanisms of collection of the products, and (iv) the hunting types. It brings out 15 spontaneous species and approximately about thirty birds species. As for the bird calls, several species of Sapotaceae which one extracts the almond of seeds are listed. At the ecological level and conservation of the vegetable biodiversity, the harvest techniques and lime conservation are described and analyzed. The Tsaaya ixeutic is an example of management of the biodiversity. It falls under the valorization of the Tsaaya knowledge related on ixeutic and the durable management of floristic diversity.

\section{INDEX}

Index géographique : Congo

Keywords : Congo, local knowledge, traditional community, floristic diversity, ethnobotany, ixeutic

Mots-clés : connaissances endogènes, sociétés traditionnelles, ethnobotanique, biodiversité végétale, ixeutique 


\section{AUTEURS}

\section{VICTOR KIMPOUNI}

École Normale Supérieure, Université Marien Ngouabi, B.P. 237, Brazzaville, Congo kimngoma@yahoo.fr

JEAN PIERRE MAKITA-MADZOU

Faculté des sciences, Université Marien Ngouabi, B.P. 69, Brazzaville, Congo

\section{ERNEST APAN}

École Normale Supérieure, Université Marien Ngouabi, B.P. 237, Brazzaville, Congo MARCEL MOTOM

École Normale Supérieure, Université Marien Ngouabi, B.P. 237, Brazzaville, Congo 